\title{
molecules
}

ISSN 1420-3049

www.mdpi.com/journal/molecules

Article

\section{Design, Synthesis and Antitumor Activity of Novel 4-Methyl-(3'S,4'S)-cis-khellactone Derivatives}

\section{Luhui Ren ${ }^{\dagger}$, Xue Du ${ }^{\dagger}$, Mengnan Hu, Chaoqun Yan, Taigang Liang * and Qingshan Li *}

School of Pharmaceutical Science, Shanxi Medical University, No 56, Xinjian Nan Road, Taiyuan 030001, Shanxi, China; E-Mails: renluhui@126.com (L.R.); duxue_1988@163.com (X.D.); hmn0624@163.com (M.H.); chaoqunchaoqunyan@163.com (C.Y.)

$\dagger$ These authors contributed equally to this work.

* Authors to whom correspondence should be addressed; E-Mails: 1taigang@gmail.com (T.L.); qingshanl@yahoo.com (Q.L.); Tel./Fax: +86-351-4690322 (T.L.).

Received: 16 February 2013; in revised form: 27 March 2013 / Accepted: 2 April 2013 / Published: 8 April 2013

\begin{abstract}
An asymmetric synthesis of a series of novel 4-methyl-(3'S,4'S)-cis-khellactone derivatives 3a-o is reported for the first time. Their structures were confirmed by ${ }^{1} \mathrm{H}-\mathrm{NMR}$, ${ }^{13} \mathrm{C}$-NMR and MS. Their cytotoxic activity was evaluated by the MTT assay against three selected human cancer cell lines: HEPG-2 (human liver carcinoma), SGC-7901 (human gastric carcinoma), LS174T (human colon carcinoma). Some compounds showed high inhibitory activity against these human cancer cell lines. Among them, compound 3a exhibited strong cytotoxicity, with $\mathrm{IC}_{50}$ values ranging from 8.51 to $29.65 \mu \mathrm{M}$. The results showed that 4-methyl-cis-khellactone derivatives with $S, S$ configuration could be a potential antitumor agents.
\end{abstract}

Keywords: 4-methyl-(3'S,4'S)-cis-khellactone derivatives; asymmetric synthesis; antitumor activity

\section{Introduction}

Khellactone coumarins, which constitute a small branch of the coumarin family, are notable because of their extensive bioactivities, including anti-HIV [1], anti-platelet aggregation [2], calcium antagonist activity [3], P-glycoprotein inhibitory ability, etc. [4,5]. Particullarly, the famous compound 
DCK [3'R,4'R-di-O-(camphanoyl-(+)-cis-khellactone] in this class, along with its derivatives, have received increasing attention due to their potent anti-HIV activity [6]. As shown in Figure 1, khellactone coumarins contain two chiral carbons, C-3' and C-4', and most of the reported compounds possess $3^{\prime} R, 4^{\prime} R$ configuration. Lots of literature has shown that the rigid stereochemistry of $3^{\prime} R$ and $4 ' R$-configured khellactone derivatives is crucial for anti-HIV activity [7-9].

Figure 1. Structures of cis-khellactone with (a) $3^{\prime} R, 4^{\prime} R$ or (b) $3 ' S, 4^{\prime} S$ configuration.

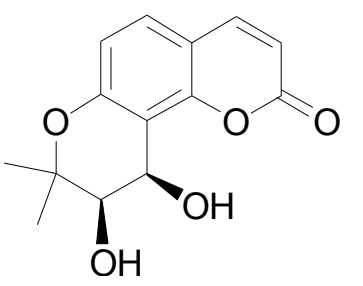

(a) $3^{\prime} R, 4^{\prime} R$ configuration

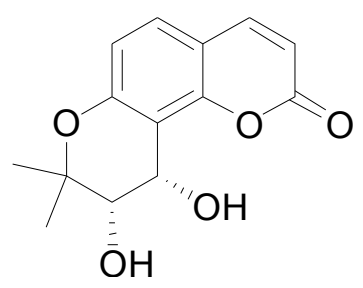

(b) 3'S,4'S configuration

Khellactone coumarins with 3 'S,4'S configuration exist mainly in the plants Peucedanum praeruptorum Dunn and Peucedanum japonicum [10]. Recently, more and more researchers have paisd close attention to the calcium antagonist activity and P-glycoprotein inhibitory ability of the (3'S,4'S)-cis-khellactone coumarins [11,12], whereas, other activities of $(3 ' S, 4 ' S)$-cis-khellactone coumarins, for example, antitumor activity, have been rarely reported.

Figure 2. Structures of 4-methyl-(3'S,4'S)-cis-khellactone derivatives 3a-0.

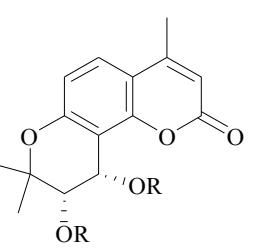

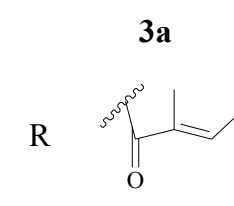
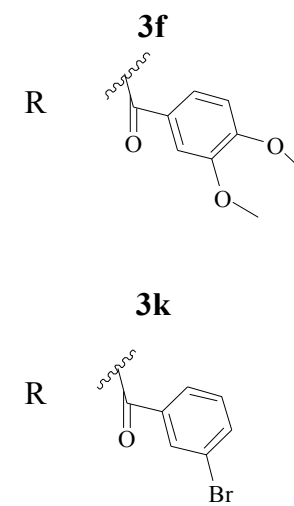

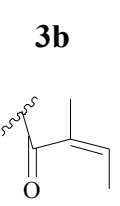

$3 g$

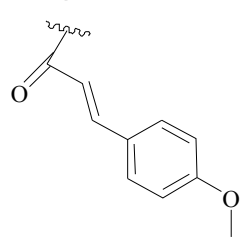

31

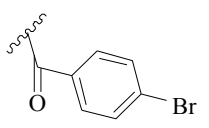

$3 \mathbf{c}$

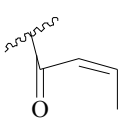

$3 \mathbf{h}$

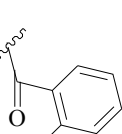

O

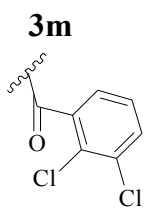

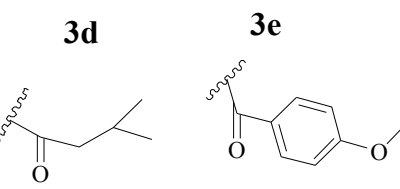

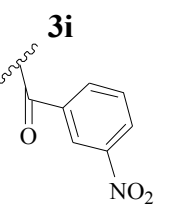

$\mathbf{3 j}$

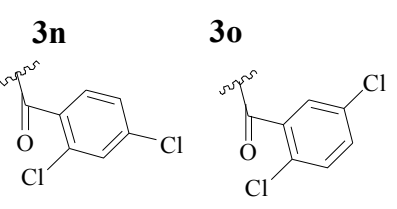

In the present study, a series of 4-methyl-(3'S,4'S)-cis-khellactone derivatives 3a-o (Figure 2) were designed and asymmetrically synthesized. Furthermore, all the synthesized compounds were screened against three cultured human cancer cell lines (HEPG-2, SGC-7901, LS174T). The results showed that 
some compounds showed potent cytotoxicity and compound 3a in particular exhibited the most significant cytotoxicity against these cancer cell lines, especially against HEPG-2 cells.

\section{Results and Discussion}

\subsection{Chemistry}

Our synthetic strategy was first to obtain 4-methylseselin (1), and then to stereoselectively synthesize 4-methyl-(-)-cis-khellactone (2) and its derivatives 3a-o. The corresponding synthetic routes are shown in Scheme 1.

Scheme 1. Synthesis of (3'S,4'S)-4-methyl-cis-khellactone derivatives 3a-0.

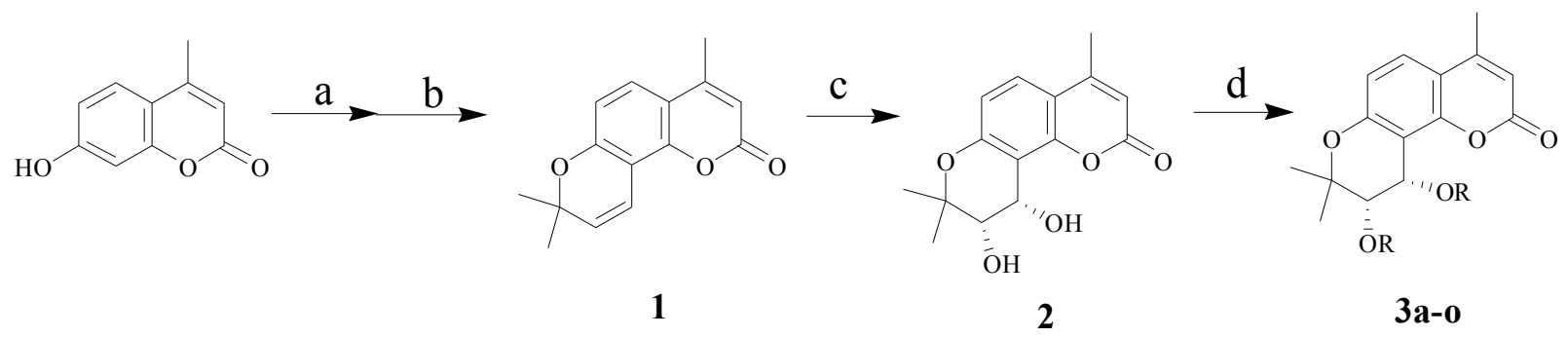

Reagent and conditions: (a) 3-chloro-3-methyl-1-butyne, $\mathrm{K}_{2} \mathrm{CO}_{3}$, $\mathrm{KI}$ in DMF, $70-80{ }^{\circ} \mathrm{C}, 3-4$ days; (b) $N, N$-diethylaniline, reflux, $15 \mathrm{~h} ;$ (c) $\mathrm{K}_{3} \mathrm{Fe}(\mathrm{CN})_{6}, \mathrm{~K}_{2} \mathrm{CO}_{3},(\mathrm{DHQD})_{2}-\mathrm{PYR}, \mathrm{K}_{2} \mathrm{OsO}_{2}(\mathrm{OH})_{4}$, methane-sulfonamide, in $\mathrm{t}-\mathrm{BuOH} / \mathrm{H}_{2} \mathrm{O}(\mathrm{v} / \mathrm{v}, 1: 1)$ at $0{ }^{\circ} \mathrm{C}, 1$ day; (d) Various organic acids, DCC, DMAP in anhydrous $\mathrm{CH}_{2} \mathrm{Cl}_{2}$, reflux.

7-Hydroxy-4-methylcoumarin, a commercially available compound, was reacted with 3-chloro-3methyl-1-butyne in DMF in the presence of anhydrous potassium carbonate and potassium iodide and then thermal rearrangement occurred in boiling diethylaniline to form 4-methylseselin (1) by following the procedures published previously $[13,14]$.

4-Methylseselin (1) was asymmetrically dihdroxylated using (DHQD) 2 -PYR (hydroquinidine 2,5diphenyl-4,6-pyrimidinediyl diether) as a chiral catalyst to give 4-methyl-(-)-cis-khellactone (2) [15]. Without further purification, compound 2 was directly esterified in anhydrous $\mathrm{CH}_{2} \mathrm{Cl}_{2}$ with various organic acids in the presence of dimethylaminopyridine (DMAP) and $N, N^{\prime}$-dicyclohexylcarbodiimide (DCC), respectively, to produce 4-methyl-cis-khellactone derivatives 3a-o [16].

For the determination of enantiomeric excess, 4-methylseselin (1) was oxidized with $\mathrm{OsO}_{4}$ to give racemic 4-methyl-cis-khellactones 2' [17] (Scheme 2). The asymmetric dihydroxylation for $\mathbf{2}$ is highly stereoselective, with good enantiomeric excess (74\% e.e.) by chiral HPLC analysis. (DHQD) $)_{2}$-PYR leads primarily to the cis-diol with $S, S$ configuration [15].

Scheme 2. Syntheses of 4-methyl-( \pm -cis-khellactone 2'.

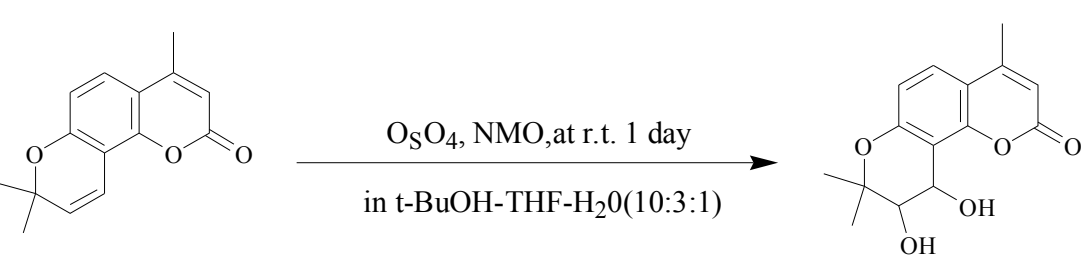




\subsection{In Vitro Biological Evaluation}

All of the 4-methyl-(3'S,4'S)-cis-khellactone derivatives were evaluated for cytotoxic activity in vitro against three human cancer cell lines (HEPG-2, SGC-7901, LS174T) using the MTT assay. The antitumor activity was indicated in terms of $\mathrm{IC}_{50}(\mu \mathrm{M})$ values and the results are presented in Table 1.

Table 1. Cytotoxic activity of the synthesized compounds against three human cancer cell lines ${ }^{\mathrm{a}}$.

\begin{tabular}{cccc}
\hline \multirow{2}{*}{ Compound } & \multicolumn{3}{c}{ IC $_{\mathbf{5 0}} \pm$ SD $(\boldsymbol{\mu M})$} \\
\cline { 2 - 4 } 3a & HEPG-2 & SGC-7901 & LS174T \\
\hline $\mathbf{3 b}$ & $8.51 \pm 3.03$ & $29.65 \pm 6.12$ & $19.14 \pm 3.68$ \\
$\mathbf{3 c}$ & $23.64 \pm 5.29$ & $59.81 \pm 9.85$ & $47.17 \pm 6.17$ \\
$\mathbf{3 d}$ & $15.62 \pm 4.15$ & $29.36 \pm 6.55$ & $20.16 \pm 4.79$ \\
$\mathbf{3 e}$ & $78.23 \pm 11.07$ & $>100$ & $>100$ \\
$\mathbf{3 f}$ & $50.92 \pm 8.01$ & $78.93 \pm 16.27$ & $54.79 \pm 9.45$ \\
$\mathbf{3 g}$ & $67.54 \pm 8.36$ & $>100$ & $55.20 \pm 12.10$ \\
$\mathbf{3 h}$ & $89.29 \pm 17.81$ & $71.30 \pm 12.76$ & $>100$ \\
$\mathbf{3 i}$ & $22.69 \pm 5.90$ & $31.14 \pm 4.32$ & $19.60 \pm 4.32$ \\
$\mathbf{3 j}$ & $>100$ & $75.23 \pm 8.09$ & $>100$ \\
$\mathbf{3 k}$ & $>100$ & $89.57 \pm 11.92$ & $91.13 \pm 13.05$ \\
$\mathbf{3 1}$ & $>100$ & $>100$ & $84.03 \pm 9.98$ \\
$\mathbf{3 m}$ & $60.92 \pm 8.73$ & $22.64 \pm 5.44$ & $38.51 \pm 4.63$ \\
$\mathbf{3 n}$ & $77.55 \pm 10.60$ & $82.09 \pm 13.76$ & $>100$ \\
$\mathbf{3 0}$ & $86.97 \pm 11.09$ & $>100$ & $>100$ \\
\hline
\end{tabular}

${ }^{a}$ The data represented the mean of three experiments in triplicate and were expressed as means \pm SD.

As shown in Table 1, some compounds showed promising anticancer activity for certain cancer cell lines in vitro and the changes of 3 ' and 4' substituents on the pyran ring had a significant influence on the cytotoxicity. For example, both compounds $\mathbf{3 a}$ and $\mathbf{3 c}$ exhibited potent inhibitory effects on the indicated cell lines, meanwhile, 3a with tigloyl group at 3' and 4' position showed the most significant cytotoxicity against the HEPG-2 cell lines, with an $\mathrm{IC}_{50}$ value of $8.51 \mu \mathrm{M}$. Compound $\mathbf{3 b}$, compared to 3a, displayed low cytotoxicity against these cell lines, especially against the SGC-7901 and LS174T cell lines. The results revealed that the $Z$ isomer of the 2-methyl-2-butenoyl group is superior to the $E$ isomer for the antitumor activity of these two compounds. Additionally, compound $\mathbf{3 h}$ with an $o$-methylbenzoyl group showed noteworthy inhibitory activity against all three human cancer cell lines among all the synthesized compounds with aromatic groups at the 3' and 4' positions. For SGC-7901 cells, the most highly active compound was $\mathbf{3 1}$ with a $p$-bromobenzoyl group, which had an $\mathrm{IC}_{50}$ value of $22.64 \mu \mathrm{M}$. The preliminary results revealed that the tigloyl group at the $3^{\prime}$ and 4 ' position on the pyran ring was favorable structural moiety to retain the anticancer activity. Further investigation to search for more potent groups is under way. 


\section{Experimental}

\subsection{General}

All chemicals were purchased from Aladdin Chemicals Co. (Shanghai, China) and Energy Chemicals Co. (Shanghai, China), whereas chiral catalyst (DHQD) 2 -PYR was obtained from Sigma-Aldrich (St. Louis, MO, USA). The reactions were monitored by thin-layer chromatography (TLC) with silica gel GF254 plates (Qingdao Jiyida Silica Reagent Manufacture, Qingdao, China), which were visualized by UV light. Melting points were measured on a XT-4 melting point apparatus (Shanghai Precision Scientific Instrument Co. Ltd., Shanghai, China) without correction. The ${ }^{1}$ H-NMR spectra were acquired on Bruker Avance $600 \mathrm{MHz}$ spectrometer (Bruker Corporation, Karlsruhe, Germany) from solutions in either deuterated chloroform or deuterated dimethylsulfoxide (DMSO- $\mathrm{d}_{6}$ ) containing tetramethylsilane as internal reference, while the ${ }^{13} \mathrm{C}-\mathrm{NMR}$ spectra were recorded at 150 MHz. ESI mass spectra were obtained on an API QTRAP 3200 LC-MS spectrometer (AB SCIEX Corporation, Boston, MA, USA). Optical rotations were measured on a Perkin-Elmer 241 polarimeter (PE Corporation, Nolwalk, CT, USA) at room temperature. The enatiomeric excesses (ee values) of the compounds were determined by Chiralpak AS-H chiral HPLC analysis by using a Shimadzu LC-10A instrument (Shimadzu, Suzhou, China), using $n$-hexane/2-propanol as eluent.

\subsection{Procedure for the Synthesis of 4-Methylseselin (1)}

To a solution of 4-methyl-7-hydroxycoumarin (1.76 g, $10 \mathrm{mmol}), \mathrm{K}_{2} \mathrm{CO}_{3}(3.45 \mathrm{~g}, 25 \mathrm{mmol})$, KI (1.66 g, $10 \mathrm{mmol})$ in DMF $(20 \mathrm{~mL})$ was added excess 3-chloro-3-methyl-1-butyne $(6 \mathrm{~mL})$, then the mixture was heated to $70-80{ }^{\circ} \mathrm{C}$ for $3-4$ days. After the solid $\mathrm{K}_{2} \mathrm{CO}_{3}$ was filtered, the brown filtrate was poured into EtOAc and washed with water three times and dried over anhydrous $\mathrm{Na}_{2} \mathrm{SO}_{4}$. The solvent was removed in vacuo. The residue, without purification, was directly heated to reflux in $20 \mathrm{~mL}$ of $\mathrm{N}, \mathrm{N}$-diethylaniline for $15 \mathrm{~h}$. The reaction mixture was cooled to room temperature, poured into EtOAc, and washed with $10 \%$ aqueous $\mathrm{HCl}$, water and brine. The organic layer was separated, and solvent was removed in vacuo. The residue was purified by column chromatography with an eluant of petroleum ether-EtOAc $=10: 1$ to afford compound 1. Molecular formula (MW): $\mathrm{C}_{15} \mathrm{H}_{14} \mathrm{O}_{3}(242.27 \mathrm{~g} / \mathrm{mol})$; white solid; 23\% Yield; mp: $139-141{ }^{\circ} \mathrm{C}$ (lit. [7]. 141-143 $\left.{ }^{\circ} \mathrm{C}\right) ;{ }^{1} \mathrm{H}-\mathrm{NMR}$ (DMSO-d 6 ) $\delta 1.43\left(6 \mathrm{H}, \mathrm{s}, 2 \times \mathrm{C}-2^{\prime}-\right.$ $\left.\mathrm{CH}_{3}\right), 2.38\left(3 \mathrm{H}, \mathrm{s}, \mathrm{C}-4-\mathrm{CH}_{3}\right), 5.93\left(1 \mathrm{H}, \mathrm{d}, J=10.04 \mathrm{~Hz}, \mathrm{H}-3{ }^{\prime}\right), 6.23(1 \mathrm{H}, \mathrm{s}, \mathrm{H}-3), 6.74(1 \mathrm{H}, \mathrm{d}, J=10.04 \mathrm{~Hz}$, H-4'), 6.82 (1H, d, $J=8.70 \mathrm{~Hz}, \mathrm{H}-6), 7.54$ (1H, d, $J=8.70 \mathrm{~Hz}, \mathrm{H}-5)$; MS (ESI) $m / z 264.9\left([\mathrm{M}+\mathrm{Na}]^{+}\right)$.

\subsection{Procedure for the Synthesis of 4-Methyl-(-)-cis-khellactone (2)}

$\mathrm{K}_{3} \mathrm{Fe}(\mathrm{CN})_{6}(246 \mathrm{mg}, 0.75 \mathrm{mmol})$ and $\mathrm{K}_{2} \mathrm{CO}_{3}(105 \mathrm{mg}, 0.75 \mathrm{mmol})$ were dissolved in $t-\mathrm{BuOH} / \mathrm{H}_{2} \mathrm{O}$ $(1: 1 \mathrm{v} / \mathrm{v}, 5 \mathrm{~mL})$ at r.t. Then, hydroquinidine 2,5-diphenyl-4,6-pyrimidinediyl diether [(DHQD $)_{2}-\mathrm{PYR}$ ], $4.4 \mathrm{mg}, 0.005 \mathrm{mmol})$ and $\mathrm{K}_{2} \mathrm{OsO}_{2}(\mathrm{OH})_{4}(2 \mathrm{mg}, 0.005 \mathrm{mmol})$ were added to the solution. The mixture was stirred for $15 \mathrm{~min}$. Then, the solution was cooled to $0{ }^{\circ} \mathrm{C}$ and methane-sulfonamide $(24 \mathrm{mg}, 0.25 \mathrm{mmol})$ added under stirring. When the solution turned from a light yellow to an orange color, compound 1 (61 mg, $0.25 \mathrm{mmol}$ ) was added. The mixture was stirred at $0{ }^{\circ} \mathrm{C}$ for 1 day. The reaction was monitored using TLC, and at completion, $\mathrm{Na}_{2} \mathrm{~S}_{2} \mathrm{O}_{5}(1 \mathrm{~g})$, water $(2.5 \mathrm{~mL})$, and $\mathrm{CH}_{2} \mathrm{Cl}_{2}(2.5 \mathrm{~mL})$ were added. After being 
stirred for $4 \mathrm{~h}$ at room temperature, the mixture was extracted with $\mathrm{CH}_{2} \mathrm{Cl}_{2}$ three times. The combined organic layer was dried over $\mathrm{Na}_{2} \mathrm{SO}_{4}$, and then solvent was removed to afford crude compound 2 . The crude compound was crystallized from petroleum ether/acetone to give pure 2. Molecular formula (MW): $\mathrm{C}_{15} \mathrm{H}_{16} \mathrm{O}_{5}(276.28 \mathrm{~g} / \mathrm{mol})$; white solid; 70\% Yield; mp: $213-215{ }^{\circ} \mathrm{C}$; $[\alpha] \mathrm{D}^{20}=-59.5(c 0.1$, DMSO); 74\% ee. HPLC: Chiralpak AS-H (hexane/i-PrOH, 90:10, flow rate $0.8 \mathrm{~mL} / \mathrm{min}$, wavelength = $323 \mathrm{~nm}), \mathrm{t}_{\mathrm{R}}($ minor $)=26.71 \mathrm{~min}, \mathrm{t}_{\mathrm{R}}($ major $)=68.88 \mathrm{~min} ;{ }^{1} \mathrm{H}-\mathrm{NMR}\left(\mathrm{DMSO}-\mathrm{d}_{6}\right) \delta 1.35\left(3 \mathrm{H}, \mathrm{s}, \mathrm{C}-2^{\prime}-\mathrm{CH}_{3}\right)$, $1.37\left(3 \mathrm{H}, \mathrm{s}, \mathrm{C}-2^{\prime}-\mathrm{CH}_{3}\right), 2.37\left(3 \mathrm{H}, \mathrm{s}, \mathrm{C}-4-\mathrm{CH}_{3}\right), 3.61\left(1 \mathrm{H}, \mathrm{br}, \mathrm{H}-4{ }^{\prime}\right), 4.90\left(1 \mathrm{H}, \mathrm{br}, \mathrm{H}-3^{\prime}\right), 5.14$ and 5.21 $($ each 1H, br, $\mathrm{OH} \times 2), 6.19(1 \mathrm{H}, \mathrm{s}, \mathrm{H}-3), 6.77(1 \mathrm{H}, \mathrm{d}, J=8.75 \mathrm{~Hz}, \mathrm{H}-6), 7.57(1 \mathrm{H}, \mathrm{d}, J=8.75 \mathrm{~Hz}, \mathrm{H}-5)$; ${ }^{13} \mathrm{C}-\mathrm{NMR}\left(\mathrm{DMSO}_{\mathrm{d}}\right.$ ) $\delta 18.17,20.98,26.86,60.23,71.23,78.68,110.55,111.71,112.56,113.42$, 125.66, 153.28, 153.52, 155.58, 160.03; MS (ESI) $m / z 277.09\left([\mathrm{M}+\mathrm{H}]^{+}\right)$.

\subsection{Procedure for the Synthesis of 4-Methyl-(3'S,4'S)-cis-Khellactone Derivatives 3a-0}

To a solution of crude compound $2(138 \mathrm{mg}, 0.5 \mathrm{mmol})$ in anhydrous $\mathrm{CH}_{2} \mathrm{Cl}_{2}(10 \mathrm{~mL})$ tiglic acid (6 mmol), angelic acid (6 mmol), crotonic acid (6 mmol), isovaleric acid (4 mmol), 4-methoxybenzoic acid (3 mmol), 3,4-dimethoxybenzoic acid ( $3 \mathrm{mmol}), 4$-methoxycinnamic acid ( $2 \mathrm{mmol}), o$-toluic acid (3 mmol), 3-nitrobenzoic acid (3 mmol), 2-bromobenzoic acid (3 mmol), 3-bromobenzoic acid (3 mmol), 4-bromobenzoic acid ( $3 \mathrm{mmol}), 2,3$-dichlorobenzoic acid ( $2 \mathrm{mmol}), 2$,4-dichlorobenzoic acid ( $2 \mathrm{mmol})$ or 2,5-dichlorobenzoic acid $(2 \mathrm{mmol})$ were added, respectively, followed by $N, N$ '-dicyclohexylcarbodiimide (412 mg, $2 \mathrm{mmol}$ ) and 4-dimethylaminopyridine ( $8 \mathrm{mg}, 0.064 \mathrm{mmol})$. The mixture was heated to reflux until the reaction was complete as monitored by TLC. After cooling to room temperature, filtered, and the filtrate was separated and purified by column chromatography (petroleum ether/acetone, 10:1) to give pure target compounds $\mathbf{3 a}-\mathbf{o}$.

(3'S,4'S)-Di-O-tigloyl-4-methyl-(+)-cis-khellactone (3a). Molecular formula (MW): $\mathrm{C}_{25} \mathrm{H}_{28} \mathrm{O}_{7}(440.49$ $\mathrm{g} / \mathrm{mol}$ ); white solid; $23 \%$ Yield; mp: $84-86{ }^{\circ} \mathrm{C}$; $[\alpha]_{D}^{20}:+3.8\left(\mathrm{c} 0.1, \mathrm{CH}_{2} \mathrm{Cl}_{2}\right) .{ }^{1} \mathrm{H}-\mathrm{NMR}\left(\mathrm{DMSO}-\mathrm{d}_{6}\right) \delta$ $1.39\left(3 \mathrm{H}, \mathrm{s}, \mathrm{C}-2^{\prime}-\mathrm{CH}_{3}\right), 1.43\left(3 \mathrm{H}, \mathrm{s}, \mathrm{C}-2^{\prime}-\mathrm{CH}_{3}\right), 1.71$ (3H, br s, H-5"), 1.72 (3H, br s, H-5"'), 1.74 (3H, br d, H-4"), 1.76 (3H, br d, H-4'"), 2.39 (3H, s, C-4-CH $), 5.32(1 \mathrm{H}, \mathrm{d}, J=4.92 \mathrm{~Hz}, \mathrm{H}-3$ '), 6.21 (1H, s, H-3), 6.50 (1H, d, $J=4.92 \mathrm{~Hz}, \mathrm{H}-4$ '), 6.66 (1H, m, H-3"), $6.72\left(1 \mathrm{H}, \mathrm{m}, \mathrm{H}-3{ }^{\prime \prime}\right), 6.94$ (1H, d, $J=8.86 \mathrm{~Hz}$, $7.72(1 \mathrm{H}, \mathrm{d}, J=8.86 \mathrm{~Hz}, \mathrm{H}-5), \mathrm{H}-6) ;{ }^{13} \mathrm{C}-\mathrm{NMR}\left(\mathrm{CDCl}_{3}\right) \delta 11.87,12.05,14.25,14.33,18.63,22.27$, 25.40, 60.64, 70.50, 77.36, 107.48, 112.06, 113.51, 113.92, 125.91, 127.96, 128.31, 137.08, 138.16, $152.04,153.46,156.47,159.72,166.50,166.56$; MS (ESI) $m / z$ 462.7,463.9 ([M+Na $\left.]^{+}\right)$.

(3'S,4'S)-Di-O-angeloyl-4-methyl-(+)-cis-khellactone (3b). Molecular formula (MW): $\mathrm{C}_{25} \mathrm{H}_{28} \mathrm{O}_{7}$ $(440.49 \mathrm{~g} / \mathrm{mol})$; white solid; $31 \%$ Yield; mp: $151-153{ }^{\circ} \mathrm{C}$; $[\alpha]_{D}^{20}:+4.2\left(\mathrm{c} 0.1, \mathrm{CH}_{2} \mathrm{Cl}_{2}\right) .{ }^{1} \mathrm{H}-\mathrm{NMR}$ $\left(\mathrm{CDCl}_{3}\right) \delta 1.42\left(3 \mathrm{H}, \mathrm{s}, \mathrm{C}-2^{\prime}-\mathrm{CH}_{3}\right), 1.48\left(3 \mathrm{H}, \mathrm{s}, \mathrm{C}-2^{\prime}-\mathrm{CH}_{3}\right), 1.73$ (3H, br d, H-4"), 1.75 (3H, br d, H-4"'), 1.78 (3H, br s, H-5"), 1.80 (3H, br s, H-5"'), $2.36\left(3 \mathrm{H}, \mathrm{s}, \mathrm{C}-4-\mathrm{CH}_{3}\right), 5.39$ (1H, d, J = $\left.4.92 \mathrm{~Hz}, \mathrm{H}-3^{\prime}\right)$, 6.08 (1H, s, H-3), 6.65 (1H, d, J= $4.92 \mathrm{~Hz}, \mathrm{H}-4$ '), 6.74 (1H, m, H-3"), 6.78 (1H, m, H-3"'), 6.83 (1H, d, $J=8.84 \mathrm{~Hz}, \mathrm{H}-6), 7.49(1 \mathrm{H}, \mathrm{d}, J=8.84 \mathrm{~Hz}, \mathrm{H}-5) ;{ }^{13} \mathrm{C}-\mathrm{NMR}\left(\mathrm{CDCl}_{3}\right) \delta 11.91,12.08,14.28,14.35$, $18.65,22.40,25.41,60.76,70.54,77.47,107.60,112.16,113.58,113.93,125.87,128.04,128.41$, $137.09,138.16,151.96,153.58,156.57,159.74,166.55,166.62$; MS (ESI) $m / z 462.9$ ([M+Na] $\left.]^{+}\right)$. 
(3'S,4'S)-Di-O-crotonoyl-4-methyl-(+)-cis-khellactone (3c). Molecular formula (MW): $\mathrm{C}_{23} \mathrm{H}_{24} \mathrm{O}_{7}$ (412.43 g/mol); white solid; 45\% Yield; mp: 63-65 ${ }^{\circ} \mathrm{C}$; $[\alpha]_{D}^{20}:+5.1\left(\mathrm{c} 0.1, \mathrm{CH}_{2} \mathrm{Cl}_{2}\right) .{ }^{1} \mathrm{H}-\mathrm{NMR}\left(\mathrm{CDCl}_{3}\right)$ $\delta 1.40\left(3 \mathrm{H}, \mathrm{s}, \mathrm{C}-2^{\prime}-\mathrm{CH}_{3}\right), 1.45\left(3 \mathrm{H}, \mathrm{s}, \mathrm{C}-2{ }^{\prime}-\mathrm{CH}_{3}\right), 1.83$ (3H, m, H-4"'), 1.85 (3H, m, H-4"), 2.35 (3H, s, C-4-CH $\left.\mathrm{CH}_{3}\right), 5.36(1 \mathrm{H}, \mathrm{d}, J=4.88 \mathrm{~Hz}, \mathrm{H}-3$ '), 5.78-5.84 (2H, m, H-2", H-2"'), 6.07 (1H, s, H-3), 6.60 (1H, d, $\left.J=4.88 \mathrm{~Hz}, \mathrm{H}-4^{\prime}\right), 6.82(1 \mathrm{H}, \mathrm{d}, J=8.84 \mathrm{~Hz}, \mathrm{H}-6), 6.90-6.98$ (2H, m, H-3", H-3"'), 7.49 (1H, d, $J=$ $8.84 \mathrm{~Hz}, \mathrm{H}-5) ;{ }^{13} \mathrm{C}-\mathrm{NMR}\left(\mathrm{CDCl}_{3}\right) \delta 17.98,18.00,18.64,22.27,25.23,60.52,70.20,77.41,107.23$, $112.05,113.50,113.94,121.79,122.03,125.95,145.18,145.96,152.09,153.41,156.46,159.73$, 164.91, 165.06; MS (ESI) $m / z$ 434.9,435.8 ([M+Na $\left.]^{+}\right)$.

(3'S,4'S)-Di-O-isovaleryloxy-4-methyl-(+)-cis-khellactone (3d). Molecular formula (MW): $\mathrm{C}_{25} \mathrm{H}_{32} \mathrm{O}_{7}$ (444.52 g/mol); light yellow oil; 52\% Yield; $[\alpha]_{D}^{20}:+4.5\left(\mathrm{c} 0.1, \mathrm{CH}_{2} \mathrm{Cl}_{2}\right) .{ }^{1} \mathrm{H}-\mathrm{NMR}\left(\mathrm{CDCl}_{3}\right) \delta 0.95-0.98$ $\left(12 \mathrm{H}, \mathrm{m}, \mathrm{CH}_{3} \times 4\right), 1.41\left(3 \mathrm{H}, \mathrm{s}, \mathrm{C}-2^{\prime}-\mathrm{CH}_{3}\right), 1.43\left(3 \mathrm{H}, \mathrm{s}, \mathrm{C}-2^{\prime}-\mathrm{CH}_{3}\right), 2.10-2.30\left(6 \mathrm{H}, \mathrm{m}, \mathrm{COCH}_{2} \times 2, \mathrm{CH}\right.$ $\times 2), 2.37\left(3 \mathrm{H}, \mathrm{s}, \mathrm{C}-4-\mathrm{CH}_{3}\right), 5.31\left(1 \mathrm{H}, \mathrm{d}, J=4.79 \mathrm{~Hz}, \mathrm{H}-3^{\prime}\right), 6.10(1 \mathrm{H}, \mathrm{s}, \mathrm{H}-3), 6.54(1 \mathrm{H}, \mathrm{d}, J=4.79 \mathrm{~Hz}$, H-4'), $6.81(1 \mathrm{H}, \mathrm{d}, J=8.85 \mathrm{~Hz}, \mathrm{H}-6), 7.49(1 \mathrm{H}, \mathrm{d}, J=8.85 \mathrm{~Hz}, \mathrm{H}-5) ;{ }^{13} \mathrm{C}-\mathrm{NMR}\left(\mathrm{CDCl}_{3}\right) \delta 18.67$, 22.36, 22.41, 25.31, 25.43, 25.47, 43.05, 43.23, 60.49, 70.37, 107.24, 112.03, 113.50, 113.94, 125.98, $152.13,153.32,156.36,159.72,171.78$; MS (ESI) $m / z 467.3\left([\mathrm{M}+\mathrm{Na}]^{+}\right)$.

(3'S,4'S)-Di-O-p-methoxybenzoyl-4-methyl-(-)-cis-khellactone (3e). Molecular formula (MW): $\mathrm{C}_{31} \mathrm{H}_{28} \mathrm{O}_{9}(544.55 \mathrm{~g} / \mathrm{mol})$; white solid; $19 \%$ Yield; mp: $177-179{ }^{\circ} \mathrm{C}$; $[\alpha]_{D}^{20}:-35.9$ (c 0.1, $\left.\mathrm{CH}_{2} \mathrm{Cl}_{2}\right)$. ${ }^{1} \mathrm{H}-\mathrm{NMR}$ $\left(\mathrm{CDCl}_{3}\right) \delta 1.49\left(3 \mathrm{H}, \mathrm{s}, \mathrm{C}-2{ }^{\prime}-\mathrm{CH}_{3}\right), 1.62\left(3 \mathrm{H}, \mathrm{s}, \mathrm{C}-2{ }^{\prime}-\mathrm{CH}_{3}\right), 2.37\left(3 \mathrm{H}, \mathrm{s}, \mathrm{C}-4-\mathrm{CH}_{3}\right), 3.82\left(3 \mathrm{H}, \mathrm{s}, \mathrm{OCH}_{3}\right)$, $3.83\left(3 \mathrm{H}, \mathrm{s}, \mathrm{OCH}_{3}\right), 5.64\left(1 \mathrm{H}, \mathrm{d}, J=4.92 \mathrm{~Hz}, \mathrm{H}-3{ }^{\prime}\right), 6.06(1 \mathrm{H}, \mathrm{s}, \mathrm{H}-3), 6.79-6.82(4 \mathrm{H}, \mathrm{m}, \mathrm{Ar}-\mathrm{H}), 6.90$ $(1 \mathrm{H}, \mathrm{d}, J=8.88 \mathrm{~Hz}, \mathrm{H}-6), 6.92(1 \mathrm{H}, \mathrm{d}, J=4.92 \mathrm{~Hz}, \mathrm{H}-4$ '), $7.54(1 \mathrm{H}, \mathrm{d}, J=8.88 \mathrm{~Hz}, \mathrm{H}-5), 7.81-7.86$ $(4 \mathrm{H}, \mathrm{m}, \mathrm{Ar}-\mathrm{H}) ;{ }^{13} \mathrm{C}-\mathrm{NMR}\left(\mathrm{CDCl}_{3}\right) \delta 18.67,22.44,25.62,55.35,55.40,61.01,70.93,77.53,107.49$, $112.25,113.50,113.62,113.99,121.83,122.40,126.09,131.84,131.89,151.88,153.63,156.60$, $159.58,163.29,163.60,164.88,164.96$; MS (ESI) $m / z 566.8\left([\mathrm{M}+\mathrm{Na}]^{+}\right)$.

(3'S,4'S)-bis-O-(3,4-Dimethoxybenzoyl)-4-methyl-(-)-cis-khellactone (3f). Molecular formula (MW): $\mathrm{C}_{33} \mathrm{H}_{32} \mathrm{O}_{11}(604.60 \mathrm{~g} / \mathrm{mol})$; white solid; $28 \%$ Yield; mp : 207-209 ${ }^{\circ} \mathrm{C}$; $[\alpha]_{D}^{20}:-10.0$ (c 0.1, $\left.\mathrm{CH}_{2} \mathrm{Cl}_{2}\right)$. ${ }^{1} \mathrm{H}-\mathrm{NMR}\left(\mathrm{CDCl}_{3}\right) \delta 1.51\left(3 \mathrm{H}, \mathrm{s}, \mathrm{C}-2^{\prime}-\mathrm{CH}_{3}\right), 1.65\left(3 \mathrm{H}, \mathrm{s}, \mathrm{C}-2{ }^{\prime}-\mathrm{CH}_{3}\right), 2.38\left(3 \mathrm{H}, \mathrm{s}, \mathrm{C}-4-\mathrm{CH}_{3}\right), 3.66(3 \mathrm{H}, \mathrm{s}$, $\left.\mathrm{OCH}_{3}\right), 3.76\left(3 \mathrm{H}, \mathrm{s}, \mathrm{OCH}_{3}\right), 3.89\left(6 \mathrm{H}, \mathrm{s}, \mathrm{OCH}_{3}\right), 5.65\left(1 \mathrm{H}, \mathrm{d}, J=4.92 \mathrm{~Hz}, \mathrm{H}-3{ }^{\prime}\right), 6.08(1 \mathrm{H}, \mathrm{s}, \mathrm{H}-3)$, $6.76(1 \mathrm{H}, \mathrm{d}, J=8.49 \mathrm{~Hz}, \mathrm{Ar}-\mathrm{H}), 6.79(1 \mathrm{H}, \mathrm{d}, J=8.49 \mathrm{~Hz}, \mathrm{Ar}-\mathrm{H}), 6.91(1 \mathrm{H}, \mathrm{d}, J=8.90 \mathrm{~Hz}, \mathrm{H}-6), 6.95$ $\left(1 \mathrm{H}, \mathrm{d}, J=4.92 \mathrm{~Hz}, \mathrm{H}-4^{\prime}\right), 7.38(1 \mathrm{H}, \mathrm{d}, J=1.92 \mathrm{~Hz}, \mathrm{Ar}-\mathrm{H}), 7.43(1 \mathrm{H}, \mathrm{d}, J=1.92 \mathrm{~Hz}, \mathrm{Ar}-\mathrm{H}), 7.50(1 \mathrm{H}$, $\mathrm{m}, \mathrm{Ar}-\mathrm{H}), 7.55(1 \mathrm{H}, \mathrm{d}, J=8.90 \mathrm{~Hz}, \mathrm{H}-5), 7.56(1 \mathrm{H}, \mathrm{m}, \mathrm{Ar}-\mathrm{H}) ;{ }^{13} \mathrm{C}-\mathrm{NMR}\left(\mathrm{CDCl}_{3}\right) \delta 18.66,22.25$, 25.73, 55.59, 55.82, 55.96, 60.98, 71.02, 77.40, 107.33, 110.22, 112.19, 112.23, 112.45, 113.60, $114.00,121.79,122.41,123.71,123.92,126.19,129.70,129.96,148.62,151.97,153.00,153.31$, $153.58,156.55,159.63,164.85,164.89$; MS (ESI) $m / z 626.8\left([\mathrm{M}+\mathrm{Na}]^{+}\right)$.

(3'S,4'S)-bis-O-p-Methoxycinnamoyl-4-methyl-(-)-cis-khellactone (3g). Molecular formula (MW): $\mathrm{C}_{35} \mathrm{H}_{32} \mathrm{O}_{9}(596.62 \mathrm{~g} / \mathrm{mol})$; white solid; 34\% Yield; mp: $120-122{ }^{\circ} \mathrm{C}$; $[\alpha]_{D}^{20}:-59.5$ (c 0.1, $\left.\mathrm{CH}_{2} \mathrm{Cl}_{2}\right)$. ${ }^{1} \mathrm{H}-\mathrm{NMR}$ $\left(\mathrm{CDCl}_{3}\right) \delta 1.47\left(3 \mathrm{H}, \mathrm{s}, \mathrm{C}-2^{\prime}-\mathrm{CH}_{3}\right), 1.56\left(3 \mathrm{H}, \mathrm{s}, \mathrm{C}-2^{\prime}-\mathrm{CH}_{3}\right), 2.37\left(3 \mathrm{H}, \mathrm{s}, \mathrm{C}-4-\mathrm{CH}_{3}\right), 3.81\left(3 \mathrm{H}, \mathrm{s}, \mathrm{OCH}_{3}\right)$, $3.82\left(3 \mathrm{H}, \mathrm{s}, \mathrm{OCH}_{3}\right), 5.51\left(1 \mathrm{H}, \mathrm{d}, J=5.00 \mathrm{~Hz}, \mathrm{H}-3^{\prime}\right), 6.10(1 \mathrm{H}, \mathrm{s}, \mathrm{H}-3), 6.31(2 \mathrm{H}, \mathrm{d}, J=15.93 \mathrm{~Hz}, 2 \times$ $\mathrm{ArCH}=), 6.77\left(1 \mathrm{H}, \mathrm{d}, J=5.00 \mathrm{~Hz}, \mathrm{H}-4{ }^{\prime}\right), 6.82-6.85(4 \mathrm{H}, \mathrm{m}, \mathrm{Ar}-\mathrm{H}), 6.88(1 \mathrm{H}, \mathrm{d}, J=8.92 \mathrm{~Hz}, \mathrm{H}-6)$, 7.37-7.41 (4H, m, Ar-H), $7.52(1 \mathrm{H}, \mathrm{d}, J=8.92 \mathrm{~Hz}, \mathrm{H}-5), 7.62(2 \mathrm{H}, \mathrm{d}, J=15.93 \mathrm{~Hz}, 2 \times-\mathrm{COCH}=)$; 
${ }^{13} \mathrm{C}-\mathrm{NMR}\left(\mathrm{CDCl}_{3}\right) \delta 18.70,22.67,25.22,55.31,55.34,60.82,70.35,77.63,107.39,112.20,113.29$, $113.43,113.65,113.98,114.16,114.25,114.53,114.99,125.96,126.98,127.26,129.82,129.96$, $132.33,132.41,145.07,145.70,151.97,153.56,156.59,159.75,161.29,161.53,165.72,166.02$; MS (ESI) $m / z 618.8\left([\mathrm{M}+\mathrm{Na}]^{+}\right)$.

(3'S,4'S)-Di-O-o-methylbenzoyl-4-methyl-(-)-cis-khellactone (3h). Molecular formula (MW): $\mathrm{C}_{31} \mathrm{H}_{28} \mathrm{O}_{7}$ $(512.55 \mathrm{~g} / \mathrm{mol})$; white solid; 47\% Yield; mp: $196-198{ }^{\circ} \mathrm{C} ;[\alpha]_{D}^{20}:-36.7$ (c $\left.0.1, \mathrm{CH}_{2} \mathrm{Cl}_{2}\right) .{ }^{1} \mathrm{H}-\mathrm{NMR}$ $\left(\mathrm{CDCl}_{3}\right) \delta 1.54\left(3 \mathrm{H}, \mathrm{s}, \mathrm{C}-2^{\prime}-\mathrm{CH}_{3}\right), 1.61\left(3 \mathrm{H}, \mathrm{s}, \mathrm{C}-2{ }^{\prime}-\mathrm{CH}_{3}\right), 2.38\left(3 \mathrm{H}, \mathrm{s}, \mathrm{C}-4-\mathrm{CH}_{3}\right), 2.43\left(3 \mathrm{H}, \mathrm{s}, \mathrm{CH}_{3}\right)$, $2.52\left(3 \mathrm{H}, \mathrm{s}, \mathrm{CH}_{3}\right), 5.66\left(1 \mathrm{H}, \mathrm{d}, J=4.95 \mathrm{~Hz}, \mathrm{H}-3{ }^{\prime}\right), 6.09(1 \mathrm{H}, \mathrm{s}, \mathrm{H}-3), 6.89(1 \mathrm{H}, \mathrm{d}, J=8.88 \mathrm{~Hz}, \mathrm{H}-6)$, $6.95\left(1 \mathrm{H}, \mathrm{d}, J=4.95 \mathrm{~Hz}, \mathrm{H}-4{ }^{\prime}\right), 7.06(1 \mathrm{H}, \mathrm{m}, \mathrm{Ar}-\mathrm{H}), 7.13(2 \mathrm{H}, \mathrm{m}, \mathrm{Ar}-\mathrm{H}), 7.20$ (1H, m, Ar-H), 7.30 $(1 \mathrm{H}, \mathrm{m}, \mathrm{Ar}-\mathrm{H}), 7.36(1 \mathrm{H}, \mathrm{m}, \mathrm{Ar}-\mathrm{H}), 7.53(1 \mathrm{H}, \mathrm{d}, J=8.88 \mathrm{~Hz}, \mathrm{H}-5), 7.65(1 \mathrm{H}, \mathrm{m}, \mathrm{Ar}-\mathrm{H}), 7.80(1 \mathrm{H}, \mathrm{m}$, $\mathrm{Ar}-\mathrm{H}) ;{ }^{13} \mathrm{C}-\mathrm{NMR}\left(\mathrm{CDCl}_{3}\right) \delta 18.69,20.92,21.56,22.48,25.61,60.99,71.01,77.43,107.41,112.18$, $113.64,114.04,125.31,125.58,126.11,128.74,129.61,129.87,130.50,131.36,131.57,131.71$, $132.24,140.53,140.94,152.04,153.55,156.54,159.66,165.96,166.25 ;$ MS (ESI) $\mathrm{m} / z \quad 534.5$ $\left([\mathrm{M}+\mathrm{Na}]^{+}\right)$.

(3'S,4'S)-Di-O-m-nitrobenzoyl-4-methyl-(-)-cis-khellactone (3i). Molecular formula (MW): $\mathrm{C}_{29} \mathrm{H}_{22} \mathrm{~N}_{2} \mathrm{O}_{11}(574.49 \mathrm{~g} / \mathrm{mol})$; white solid; 34\% Yield; mp: 187-189 ${ }^{\circ} \mathrm{C}$; $[\alpha]_{D}^{20}$ : -10.0 (c $\left.0.1, \mathrm{CH}_{2} \mathrm{Cl}_{2}\right)$. ${ }^{1} \mathrm{H}-\mathrm{NMR}\left(\mathrm{CDCl}_{3}\right) \delta 1.54\left(3 \mathrm{H}, \mathrm{s}, \mathrm{C}-2^{\prime}-\mathrm{CH}_{3}\right), 1.65\left(3 \mathrm{H}, \mathrm{s}, \mathrm{C}-2^{\prime}-\mathrm{CH}_{3}\right), 2.40\left(3 \mathrm{H}, \mathrm{s}, \mathrm{C}-4-\mathrm{CH}_{3}\right), 5.74(1 \mathrm{H}$, d, $\left.J=4.95 \mathrm{~Hz}, \mathrm{H}-3^{\prime}\right), 6.07$ (1H, s, H-3), 6.96 (1H, d, $\left.J=8.93 \mathrm{~Hz}, \mathrm{H}-6\right), 6.98$ (1H, d, $\left.J=4.95 \mathrm{~Hz}, \mathrm{H}-4^{\prime}\right)$, 7.58-7.63 (2H, m, Ar-H), $7.61(1 \mathrm{H}, \mathrm{d}, J=8.93 \mathrm{~Hz}, \mathrm{H}-5), 8.27$ (2H, m, Ar-H), 8.39 (2H, m, Ar-H), $8.56(1 \mathrm{H}, \mathrm{m}, \mathrm{Ar}-\mathrm{H}), 8.60(1 \mathrm{H}, \mathrm{m}, \mathrm{Ar}-\mathrm{H}) ;{ }^{13} \mathrm{C}-\mathrm{NMR}\left(\mathrm{CDCl}_{3}\right) \delta 18.78,22.78,25.04,62.48,71.82,77.30$, $105.94,112.22,113.93,114.21,124.30,124.46,126.76,127.69,128.00,129.78,129.91,130.71$, $131.07,135.41,135.71,148.07,148.21,152.29,153.42,156.40,159.47,163.46,163.57$; MS (ESI) $m / z$ $596.7\left([\mathrm{M}+\mathrm{Na}]^{+}\right)$.

(3'S,4'S)-Di-O-o-bromobenzoyl-4-methyl-(-)-cis-khellactone (3j). Molecular formula (MW): $\mathrm{C}_{29} \mathrm{H}_{22} \mathrm{Br}_{2} \mathrm{O}_{7}(642.29 \mathrm{~g} / \mathrm{mol})$; white solid; $42 \%$ Yield; mp: $178-180{ }^{\circ} \mathrm{C}$; $[\alpha]_{D}^{20}$ : $-62.1\left(\mathrm{c} 0.1, \mathrm{CH}_{2} \mathrm{Cl}_{2}\right)$. ${ }^{1} \mathrm{H}-\mathrm{NMR}\left(600 \mathrm{MHz}, \mathrm{CDCl}_{3}\right) \delta 1.59\left(3 \mathrm{H}, \mathrm{s}, \mathrm{C}-2{ }^{\prime}-\mathrm{CH}_{3}\right), 1.60\left(3 \mathrm{H}, \mathrm{s}, \mathrm{C}-2{ }^{\prime}-\mathrm{CH}_{3}\right), 2.39\left(3 \mathrm{H}, \mathrm{s}, \mathrm{C}-4-\mathrm{CH}_{3}\right)$, $5.73\left(1 \mathrm{H}, \mathrm{d}, J=4.51 \mathrm{~Hz}, \mathrm{H}-3{ }^{\prime}\right), 6.12(1 \mathrm{H}, \mathrm{s}, \mathrm{H}-3), 6.87(1 \mathrm{H}, \mathrm{d}, J=8.57 \mathrm{~Hz}, \mathrm{H}-6), 6.94(1 \mathrm{H}, \mathrm{d}, J=4.51 \mathrm{~Hz}$, H-4'), $7.22(1 \mathrm{H}, \mathrm{d}, J=7.31 \mathrm{~Hz}, \mathrm{Ar}-\mathrm{H}), 7.25(1 \mathrm{H}, \mathrm{d}, J=7.31 \mathrm{~Hz}, \mathrm{Ar}-\mathrm{H}), 7.31(2 \mathrm{H}, \mathrm{m}, \mathrm{Ar}-\mathrm{H}), 7.53$ (2H, m, Ar-H), $7.61(1 \mathrm{H}, \mathrm{d}, J=7.57 \mathrm{~Hz}, \mathrm{Ar}-\mathrm{H}), 7.71(1 \mathrm{H}, \mathrm{d}, J=7.57 \mathrm{~Hz}, \mathrm{Ar}-\mathrm{H}), 7.84(1 \mathrm{H}, \mathrm{d}, J=8.57 \mathrm{~Hz}$, $\mathrm{H}-5) ;{ }^{13} \mathrm{C}-\mathrm{NMR}\left(150 \mathrm{MHz}, \mathrm{CDCl}_{3}\right) \delta 18.73,22.25,25.97,62.13,72.11,77.45,106.84,112.19,113.61$, $114.12,120.96,121.41,126.42,127.06,127.20,131.03,131.77,132.05,132.10,132.68,132.76$, 133.74, 134.08, 152.16, 153.48, 156.43, 159.61, 165.20, 165.67; MS (ESI) $m / z$ 662.0, 664.0, 666.5 $\left([\mathrm{M}+\mathrm{Na}]^{+}\right)$.

(3'S,4'S)-Di-O-m-bromobenzoyl-4-methyl-(-)-cis-khellactone (3k). Molecular formula (MW): $\mathrm{C}_{29} \mathrm{H}_{22} \mathrm{Br}_{2} \mathrm{O}_{7}(642.29 \mathrm{~g} / \mathrm{mol})$; white solid; 36\% Yield; mp: $201-203{ }^{\circ} \mathrm{C}$; $[\alpha]_{D}^{20}:-17.8\left(\mathrm{c} 0.1, \mathrm{CH}_{2} \mathrm{Cl}_{2}\right)$. ${ }^{1} \mathrm{H}-\mathrm{NMR}\left(\mathrm{CDCl}_{3}\right) \delta 1.51\left(3 \mathrm{H}, \mathrm{s}, \mathrm{C}-2^{\prime}-\mathrm{CH}_{3}\right), 1.63\left(3 \mathrm{H}, \mathrm{s}, \mathrm{C}-2^{\prime}-\mathrm{CH}_{3}\right), 2.39\left(3 \mathrm{H}, \mathrm{s}, \mathrm{C}-4-\mathrm{CH}_{3}\right), 5.66(1 \mathrm{H}$, $\left.\mathrm{d}, J=5.00 \mathrm{~Hz}, \mathrm{H}-3^{\prime}\right), 6.09(1 \mathrm{H}, \mathrm{s}, \mathrm{H}-3), 6.92(1 \mathrm{H}, \mathrm{d}, J=8.52 \mathrm{~Hz}, \mathrm{H}-6), 6.93\left(1 \mathrm{H}, \mathrm{d}, J=5.00 \mathrm{~Hz}, \mathrm{H}-4^{\prime}\right)$, 7.24-7.27 (2H, m, Ar-H), 7.58 (1H, d, $J=8.52 \mathrm{~Hz}, \mathrm{H}-5), 7.63-7.68$ (2H, m, Ar-H), 7.81-7.86 (2H, m, $\mathrm{Ar}-\mathrm{H}), 7.95(2 \mathrm{H}, \mathrm{m}, \mathrm{Ar}-\mathrm{H}) ;{ }^{13} \mathrm{C}-\mathrm{NMR}\left(\mathrm{CDCl}_{3}\right) \delta 18.70,22.49,25.36,61.70,71.46,77.31,106.59$, 
$112.31,113.80,114.08,122.38,122.53,126.47,128.35,128.46,129.96,130.02,131.08,131.54$, $132.55,132.64,135.97,136.37,152.01,153.52,156.45,159.46,164.04,164.07$; MS (ESI) $\mathrm{m} / z$ 662.1, $664.3,666.5\left([\mathrm{M}+\mathrm{Na}]^{+}\right)$.

(3'S,4'S)-Di-O-p-bromobenzoyl-4-methyl-(-)-cis-khellactone (31). Molecular formula (MW): $\mathrm{C}_{29} \mathrm{H}_{22} \mathrm{Br}_{2} \mathrm{O}_{7}(642.29 \mathrm{~g} / \mathrm{mol})$; white solid; 40\% Yield; mp: 192-194 ${ }^{\circ} \mathrm{C}$; $[\alpha]_{D}^{20}:-20.0$ (c $0.1, \mathrm{CH}_{2} \mathrm{Cl}_{2}$ ). ${ }^{1} \mathrm{H}-\mathrm{NMR}\left(\mathrm{CDCl}_{3}\right) \delta 1.50\left(3 \mathrm{H}, \mathrm{s}, \mathrm{C}-2^{\prime}-\mathrm{CH}_{3}\right), 1.60\left(3 \mathrm{H}, \mathrm{s}, \mathrm{C}-2^{\prime}-\mathrm{CH}_{3}\right), 2.38\left(3 \mathrm{H}, \mathrm{s}, \mathrm{C}-4-\mathrm{CH}_{3}\right), 5.64(1 \mathrm{H}$, d, $\left.J=4.82 \mathrm{~Hz}, \mathrm{H}-3^{\prime}\right), 6.08(1 \mathrm{H}, \mathrm{s}, \mathrm{H}-3), 6.91(1 \mathrm{H}, \mathrm{d}, J=8.98 \mathrm{~Hz}, \mathrm{H}-6), 6.92\left(1 \mathrm{H}, \mathrm{d}, J=4.82 \mathrm{~Hz}, \mathrm{H}-4^{\prime}\right)$, 7.49 (4H, m, Ar-H), $7.56(1 \mathrm{H}, \mathrm{d}, J=8.98 \mathrm{~Hz}, \mathrm{H}-5), 7.72(4 \mathrm{H}, \mathrm{m}, \mathrm{Ar}-\mathrm{H}) ;{ }^{13} \mathrm{C}-\mathrm{NMR}\left(\mathrm{CDCl}_{3}\right) \delta 18.70$, $22.49,25.36,61.68,71.35,77.35,106.71,112.24,113.76,114.13,126.41,128.10,128.21,128.52$, $128.62,129.62,131.17,131.57,131.68,131.81,132.15,152.17,153.48,156.49,159.67,164.60$, 164.69; MS (ESI) $m / z$ 662.6, 664.5, $666.5\left([\mathrm{M}+\mathrm{Na}]^{+}\right)$.

(3'S,4'S)-bis-O-(2,3-dichlorobenzoyl)-4-methyl-(-)-cis-khellactone (3m). Molecular formula (MW): $\mathrm{C}_{29} \mathrm{H}_{20} \mathrm{Cl}_{4} \mathrm{O}_{7}(622.28 \mathrm{~g} / \mathrm{mol})$; white solid; $29 \%$ Yield; mp: $197-199{ }^{\circ} \mathrm{C}$; $[\alpha]_{D}^{20}:-34.6\left(\mathrm{c} 0.1, \mathrm{CH}_{2} \mathrm{Cl}_{2}\right)$. ${ }^{1} \mathrm{H}-\mathrm{NMR}\left(\mathrm{CDCl}_{3}\right) \delta 1.55\left(3 \mathrm{H}, \mathrm{s}, \mathrm{C}-2^{\prime}-\mathrm{CH}_{3}\right), 1.61\left(3 \mathrm{H}, \mathrm{s}, \mathrm{C}-2^{\prime}-\mathrm{CH}_{3}\right), 2.40\left(3 \mathrm{H}, \mathrm{s}, \mathrm{C}-4-\mathrm{CH}_{3}\right), 5.71(1 \mathrm{H}$, d, $\left.J=4.88 \mathrm{~Hz}, \mathrm{H}-3^{\prime}\right), 6.14(1 \mathrm{H}, \mathrm{s}, \mathrm{H}-3), 6.88(1 \mathrm{H}, \mathrm{d}, J=8.85 \mathrm{~Hz}, \mathrm{H}-6), 6.92\left(1 \mathrm{H}, \mathrm{d}, J=4.88 \mathrm{~Hz}, \mathrm{H}-4^{\prime}\right)$, $7.18(1 \mathrm{H}, \mathrm{m}, \mathrm{Ar}-\mathrm{H}), 7.26(1 \mathrm{H}, \mathrm{m}, \mathrm{Ar}-\mathrm{H}), 7.49(1 \mathrm{H}, \mathrm{m}, \mathrm{Ar}-\mathrm{H}), 7.55(1 \mathrm{H}, \mathrm{d}, J=8.85 \mathrm{~Hz}, \mathrm{H}-5), 7.58(1 \mathrm{H}$, m, Ar-H), $7.61(1 \mathrm{H}, \mathrm{m}, \mathrm{Ar}-\mathrm{H}), 7.72(1 \mathrm{H}, \mathrm{m}, \mathrm{Ar}-\mathrm{H}) ;{ }^{13} \mathrm{C}-\mathrm{NMR}\left(\mathrm{CDCl}_{3}\right) \delta$ 18.73, 21.90, 25.99, 62.44, $72.49,77.28,106.58,112.19,113.67,114.23,126.60,127.32,127.36,128.63,129.67,130.62,131.19$, $132.41,132.60,133.29,133.45,133.95,134.33,152.33,153.42,156.38,159.66,164.55,165.12$; MS (ESI) $m / z 642.1,644.2\left([\mathrm{M}+\mathrm{Na}]^{+}\right)$.

(3'S,4'S)-bis-O-(2,4-dichlorobenzoyl)-4-methyl-(-)-cis-khellactone (3n). Molecular formula (MW): $\mathrm{C}_{29} \mathrm{H}_{20} \mathrm{Cl}_{4} \mathrm{O}_{7}(622.28 \mathrm{~g} / \mathrm{mol})$; white solid; 41\% Yield; mp: 140-142 ${ }^{\circ} \mathrm{C}$; $[\alpha]_{D}^{20}:-73.1$ (c $\left.0.1, \mathrm{CH}_{2} \mathrm{Cl}_{2}\right)$. ${ }^{1} \mathrm{H}-\mathrm{NMR}\left(\mathrm{CDCl}_{3}\right) \delta 1.56\left(3 \mathrm{H}, \mathrm{s}, \mathrm{C}-2^{\prime}-\mathrm{CH}_{3}\right), 1.57\left(3 \mathrm{H}, \mathrm{s}, \mathrm{C}-2^{\prime}-\mathrm{CH}_{3}\right), 2.39\left(3 \mathrm{H}, \mathrm{s}, \mathrm{C}-4-\mathrm{CH}_{3}\right), 5.69(1 \mathrm{H}$, d, $\left.J=5.18 \mathrm{~Hz}, \mathrm{H}-3^{\prime}\right), 6.12(1 \mathrm{H}, \mathrm{s}, \mathrm{H}-3), 6.88\left(1 \mathrm{H}, \mathrm{d}, J=5.18 \mathrm{~Hz}, \mathrm{H}-4{ }^{\prime}\right), 6.89(1 \mathrm{H}, \mathrm{d}, J=8.95 \mathrm{~Hz}, \mathrm{H}-6)$, $7.21(1 \mathrm{H}, \mathrm{m}, \mathrm{Ar}-\mathrm{H}), 7.27(1 \mathrm{H}, \mathrm{m}, \mathrm{Ar}-\mathrm{H}), 7.37(1 \mathrm{H}, J=1.92 \mathrm{~Hz}, \mathrm{Ar}-\mathrm{H}), 7.44(1 \mathrm{H}, J=1.92 \mathrm{~Hz}, \mathrm{Ar}-\mathrm{H})$, $7.55(1 \mathrm{H}, \mathrm{d}, J=8.95 \mathrm{~Hz}, \mathrm{H}-5), 7.71(1 \mathrm{H}, \mathrm{d}, J=8.45 \mathrm{~Hz}, \mathrm{Ar}-\mathrm{H}), 7.82(1 \mathrm{H}, \mathrm{d}, J=8.45 \mathrm{~Hz}, \mathrm{Ar}-\mathrm{H}) ;{ }^{13} \mathrm{C}-\mathrm{NMR}$ $\left(\mathrm{CDCl}_{3}\right) \delta 18.72,22.23,25.65,62.28,72.12,77.28,106.57,112.19,113.67,114.14,126.50,127.02$, $127.12,127.69,128.69,130.51,130.89,132.24,133.11,134.21,134.73,138.00,138.71,152.22$, $153.44,156.38,159.53,163.86,164.38 ; \mathrm{MS}(\mathrm{ESI}) \mathrm{m} / z$ 642.0, $644.2\left([\mathrm{M}+\mathrm{Na}]^{+}\right)$.

(3'S,4'S)-bis-O-(2,5-dichlorobenzoyl)-4-methyl-(-)-cis-khellactone (3o). Molecular formula (MW): $\mathrm{C}_{29} \mathrm{H}_{20} \mathrm{Cl}_{4} \mathrm{O}_{7}(622.28 \mathrm{~g} / \mathrm{mol})$; white solid; $28 \%$ Yield; mp: $116-118{ }^{\circ} \mathrm{C}$; $[\alpha]_{D}^{20}:-71.2\left(\mathrm{c} 0.1, \mathrm{CH}_{2} \mathrm{Cl}_{2}\right)$. ${ }^{1} \mathrm{H}-\mathrm{NMR}\left(\mathrm{CDCl}_{3}\right) \delta 1.58\left(6 \mathrm{H}, \mathrm{s}, 2 \times \mathrm{C}^{2} 2^{\prime}-\mathrm{CH}_{3}\right), 2.40\left(3 \mathrm{H}, \mathrm{s}, \mathrm{C}-4-\mathrm{CH}_{3}\right), 5.69\left(1 \mathrm{H}, \mathrm{d}, J=4.95 \mathrm{~Hz}, \mathrm{H}-3{ }^{\prime}\right)$, $6.14(1 \mathrm{H}, \mathrm{s}, \mathrm{H}-3), 6.90(1 \mathrm{H}, \mathrm{d}, J=8.88 \mathrm{~Hz}, \mathrm{H}-6), 6.91\left(1 \mathrm{H}, \mathrm{d}, J=4.95 \mathrm{~Hz}, \mathrm{H}-4{ }^{\prime}\right), 7.29-7.40$ (4H, m, Ar-H), 7.57 (1H, d, $J=8.88 \mathrm{~Hz}, \mathrm{H}-5), 7.72(1 \mathrm{H}, \mathrm{d}, J=2.30 \mathrm{~Hz}, \mathrm{Ar}-\mathrm{H}), 7.81(1 \mathrm{H}, \mathrm{d}, J=2.30 \mathrm{~Hz}, \mathrm{Ar}-\mathrm{H})$; ${ }^{13} \mathrm{C}-\mathrm{NMR}\left(\mathrm{CDCl}_{3}\right) \delta 18.73,22.17,25.70,62.32,72.32,106.38,112.28,113.73,114.12,126.61$, $130.61,131.08,131.27,131.72,131.75,131.87,131.92,132.13,132.34,132.60,132.82,152.13$, $153.45,156.33,159.44,163.56,163.87$; MS (ESI) $m / z 642.0,644.2\left([\mathrm{M}+\mathrm{Na}]^{+}\right)$. 


\subsection{Procedure for the Synthesis of 4-methyl-(土)-cis-Khellactone (2')}

Compound 1 (0.242 g, $1 \mathrm{mmol}$ ) was added to a solution of osmium tetroxide (10 $\mathrm{mg}, 0.04 \mathrm{mmol})$ and $N$-methylmorpholine- $N$-oxide monohydrate $(0.129 \mathrm{~g}, 1.1 \mathrm{mmol})$ in $t$-BuOH-THF- $\mathrm{H}_{2} \mathrm{O}(10: 3: 1,10 \mathrm{~mL})$ and the mixture was stirred for 1 day at r.t. Saturated $\mathrm{NaHSO}_{3}$ solution $(80 \mathrm{~mL})$ was added, the mixture kept stirring for $2 \mathrm{~h}$ and extracted with $\mathrm{CH}_{2} \mathrm{Cl}_{2}(2 \times 40 \mathrm{~mL})$ and purified by column chromatography (petroleum ether/acetone, 5:1) to afford the pure compound 2'. Molecular formula (MW) : $\mathrm{C}_{15} \mathrm{H}_{16} \mathrm{O}_{5}(276.28 \mathrm{~g} / \mathrm{mol})$; white solid; 56\% Yield; MS (ESI) $m / z 277.09$ ([M+H] $]^{+}$).

\subsection{In Vitro Biological Evaluation}

All the cell lines were cultured in Dulbecco's modified Eagle's medium (DMEM), supplemented with $10 \%(\mathrm{v} / \mathrm{v})$ heat-inactivated fetal bovine serum (FBS), 100 units $/ \mathrm{mL}$ penicillin, and $100 \mu \mathrm{g} / \mathrm{mL}$ streptomycin in a humidified incubator aerated with $5 \% \mathrm{CO}_{2}$ at $37{ }^{\circ} \mathrm{C}$. The MTT assay was used to evaluate the cytotoxic activities in vitro. Briefly, cells were harvested during logarithmic growth phase and seeded in 96 well plates at a density of $2 \times 10^{4}$ cells per well. After $24 \mathrm{~h}$ incubation at $37{ }^{\circ} \mathrm{C}$ in $5 \%$ $\mathrm{CO}_{2}$ atmosphere, cells were exposed to title compounds at concentrations from 5 to $200 \mu \mathrm{M}$ for $48 \mathrm{~h}$. Subsequently, MTT ( $25 \mu \mathrm{L}, 5 \mathrm{mg} / \mathrm{mL})$ was added to each well and the plates were incubated for a further $4 \mathrm{~h}$ at $37^{\circ} \mathrm{C}$. Then, the media were removed from all the wells and the resultant MTT formazan was solubilized in DMSO $(200 \mu \mathrm{L})$. The absorbance values at $490 \mathrm{~nm}$ were measured using a microplate reader (Model 680, Bio-Rad, Hercules, CA, USA). The $\mathrm{IC}_{50}$ was calculated from the semilogarithmic dose-response curves.

\section{Conclusions}

In conclusion, a series of novel 4-methyl-(3'S,4'S)-cis-khellactone derivatives were designed and asymmetrically synthesized. Antitumor evaluation indicated that some of these new compounds displayed high inhibitory activity against three human cancer cell lines. Among them, compound 3a with a tigloyl group at the $3^{\prime}, 4^{\prime}$ positions on the pyran ring exhibited the most potent cytotoxic activity and these results could be useful for the further modification to obtain more potent antitumor agents.

\section{Acknowledgments}

Financial supports from the National Natural Science Foundation of China (No. 81101687), the Natural Science Foundation of Shanxi Province (No. 2008021048), the Program for the Top Science and Technology Innovation Teams of Higher Learning Institutions of Shanxi Province, the Program for the Top Young and Middle-aged Innovative Talents of Higher Learning Institutions of Shanxi Province are gratefully acknowledged.

\section{Conflict of Interest}

The authors declare no conflict of interest. 


\section{References}

1. Lee, T.T.; Kashiwada, Y.; Huang, L.; Snider, J.; Cosentino, M.; Lee, K.H. Suksdorfin: An antiHIV principle from Lomutium suksdorfii, its structure-activity correlation with related coumarins, and synergistic effects with anti-AIDS nucleosides. Bioorgan. Med. Chem. 1994, 2, 1051-1056.

2. Tsai, I.L.; Wun, M.F.; Teng, C.M.; Ishikawa, T.; Chen, I.S. Anti-platelet aggregation constituents from Formosan Toddalia asiatica. Phytochemistry 1998, 48, 1377-1382.

3. Kozawa, T.; Sakai, K.; Uchida, M.; Okuyama, T.; Shibata, S. Calcium antagonistic action of a coumarin isolated from"Qian-Hu”, A Chinese traditional medicine. J. Pharm. Pharmacol. 1981, $33,317-320$.

4. Wu, J.Y.; Fong, W.F.; Zhang, J.X.; Leung, C.H.; Kwong, H.L.; Yang, M.S.; Li, D.; Cheung, H.Y. Reversal of multidrug resistance in cancer cells by pyranocoumarins isolated from Radix Peucedani. Eur. J. Pharmacol. 2003, 473, 9-17.

5. Shen, X.L.; Chen, G.Y.; Zhu, G.Y.; Cai, J.Z.; Wang, L.; Hu, Y.J.; Fong, W.F. 3'-O, 4'-O-aromatic acyl substituted 7,8-pyranocoumarins: a new class of P-glycoprotein modulators. J. Pharm. Pharmacol. 2012, 64, 90-100.

6. Huang, L.; Kashiwada, Y.; Cosentino, M.L.; Fan, S.; Chen, C.H.; McPhai1, A.T.; Fujioka, T.; Mihashi, K.; Lee, K.H. Anti-AIDS agents. 15. synthesis and anti-HIV activity of dihydroseselins and related analogs. J. Med. Chem. 1994, 37, 3947-3955.

7. Xie, L.; Takeuchi, Y.; Cosentino, M.L.; Lee, K.H. Anti-AIDS agents. 37.synthesis and structure activity relationships of $\left(3^{\prime} R, 4^{\prime} R\right)-(+)$-cis-khellactone derivatives as novel potent anti-HIV agents. J. Med. Chem. 1999, 42, 2662-2672.

8. Takeuchi, Y.; Xie, L.; Cosentino, M.L.; Lee, K.H. Anti-AIDS agents-XXVIII synthesis and anti-HIV activity of methoxy substituted 3',4'-Di-O-(-)-Camphanoyl-(+)-cis-khellactone (DCK) Analogues. Bioorg. Med. Chem. Lett. 1997, 7, 2573-2578.

9. Xie, L.; Yu, D.L.; Wild, C.; Allaway, G.; Turpin, J.; Smith, P.C.; Lee, K.H. Anti-AIDS agents. 52 . synthesis and anti-HIV activity of hydroxymethyl $\left(3^{\prime} R, 4^{\prime} R\right)-3^{\prime}, 4^{\prime}-\mathrm{Di}-O-(\mathrm{S})$-camphanoyl-(+)cis-khellactone derivatives. J. Med. Chem. 2004, 47, 756-760.

10. Chen, Z.X.; Huang, B.S.; She, Q.L.; Zeng, G.F. The chemical constituents of Bai-hua-qian-hu, the root of Peucedanum praeruptorum Dunn, (umbelliferae)-four new coumarins. Acta Pharm. Sin. 1979, 14, 486-495.

11. Shen, X.L.; Chen, G.Y.; Zhu, G.Y.; Fong, W.F. ( \pm )- 3'-O, 4'-O-dicynnamoyl-cis-khellactone, a derivative of $( \pm)$-praeruptorin $\mathrm{A}$, reverses P-glycoprotein mediated multidrug resistance in cancer cells. Bioorgan. Med. Chem. 2006, 14, 7138-7145.

12. Fong, W.F.; Shen, X.L.; Globisch, C.; Wiese, M.; Chen, G.Y.; Zhu, G.Y.; Yu, Z.L.; Tse A.K.; $\mathrm{Hu}$, Y.J. Methoxylation of 3',4'-aromatic side chains improves P-glycoprotein inhibitory and multidrug resistance reversal activities of 7,8-pyranocoumarin against cancer cells. Bioorgan. Med. Chem. 2008, 16, 3694-3703.

13. Hlubucek, J.; Ritchie, E.; Taylor, W.C. Synthesis of 2,2-dimethylchromenes. Aust. J. Chem. 1971, 24, 2347-2354.

14. Murray, R.D.; Ballantyne, M.M.; Mathai, K.P. Claisen rearrangements III. Convenient synthesis of the coumarins, osthenol, demethylsuberosin and coumurrayin. Tetrahedron 1971, 27, 1247-1251. 
15. Xie, L.; Crimmins, M.T.; Lee, K.H. Asymmetric synthesis of 3',4'-Di-O-(-)-camphanoyl-(+)-ciskhellactone (DCK), A potent anti-HIV agent. Tetrahedron Lett. 1995, 36, 4529-4532.

16. Wu, X.L.; Kong, L.Y.; Min, Z.D. Studies on structure modification of (+)-praeruptorinA. Acta Pharm. Sin. 2002, 37, 527-534.

17. Ternbe, S.B.; Bhedi, D.N.; Souza, N.J.; Rupp, R.H. Synthesis of $( \pm)$ praeruptorin A and related khellactone derivatives. Heterocycles 1987, 26, 1239-1249.

Sample Availability: Samples of the compounds are available from the authors.

(C) 2013 by the authors; licensee MDPI, Basel, Switzerland. This article is an open access article distributed under the terms and conditions of the Creative Commons Attribution license (http://creativecommons.org/licenses/by/3.0/). 